\title{
Variable Step Random Walks and Self-Similar Distributions*
}

\author{
Gemunu H. Gunaratne, ${ }^{1,2}$ Joseph L. McCauley, ${ }^{1}$ Matthew Nicol, ${ }^{3}$ and \\ Andrei Törö ${ }^{3,4}$
}

Received December 13, 2004; accepted March 30, 2005

\begin{abstract}
We study a scenario under which variable step random walks give anomalous statistics. We begin by analyzing the Martingale Central Limit Theorem to find a sufficient condition for the limit distribution to be non-Gaussian. We study the case when the scaling index $\zeta$ is $\frac{1}{2}$. For corresponding continuous time processes, it is shown that the probability density function $W(x ; t)$ satisfies the Fokker-Planck equation. Possible forms for the diffusion coefficient are given, and related to $W(x, t)$. Finally, we show how a time-series can be used to distinguish between these variable diffusion processes and Lévy dynamics.
\end{abstract}

KEY WORDS: Martingale process; central limit theorem; non-Gaussian distributions; Fokker-Planck equation.

\section{INTRODUCTION}

Under which conditions can statistics of stochastic processes be anomalous? Such statistics have been observed in temperature and longitudinal velocity fluctuations in highly turbulent fluid flows, ${ }^{(1-5)}$ instantaneous velocities of gusting winds ${ }^{(6)}$ and price variations in financial markets. ${ }^{(7-12)}$ Furthermore, in the case of financial markets, it has been noted

\footnotetext{
*It is a great pleasure to dedicate this paper to Mitchell Feigenbaum on the occasion of his 60th birthday. Mitchell's outlook on science, arts, and philosopy has been a source of inspiration for GHG for over 20 years.

${ }^{1}$ Department of Physics, University of Houston, Houston, TX 77204, USA; e-mail: gemunu.gunarathe@mail.un.edu

${ }^{2}$ Institute for Fundamental Studies, Hantana, Sri Lanka.

${ }^{3}$ Department of Mathematics, University of Houston, Houston, TX 77204.

${ }^{4}$ Institute of Mathematics of the Romanian Academy, Bucharest, Romania.
} 
that the probability density functions exhibit self-similarity. ${ }^{(7,8)}$ In prior work, Lévy statistics ${ }^{(13-15)}$ and hierarchical processes ${ }^{(16)}$ have been suggested as possible causes of anomalous behavior. In this paper we suggest an alternative scenario where, in contrast to Lévi processes, stochastic increments have uniformly bounded variance and are not independent.

General conditions for the validity of the Central Limit Theorem (CLT) have been given for martingales, which are defined in Section 2. We discuss how the limit distribution can fail to be Gaussian, and provide a set of conditions that give non-Gaussian statistics. In Section 3, we argue that the corresponding probability density $W(x ; t)$ for continuous time processes satisfies the Fokker-Planck equation ${ }^{(17,18)}$ and scales with index $\zeta\left(=\frac{1}{2}\right)$; i.e., $W(x ; t)=\frac{1}{\sqrt{t}} F(u)$, where $u=\frac{x}{\sqrt{t}}$. By using the FokkerPlanck equation, we show that the diffusion coefficient $D(x ; t)$ for the process takes a specific form. We show that a reduction of $D(x ; t)$ to a form $D(u)$ preserves all statistical features of the stochastic process. Given $D(u)$, we then provide an explicit expression for $F(u)$. As examples, we provide forms for $D(u)$ that give exponential and power-law distributions for $F(u)$. In Section 4, we provide a criterion that can be used to distinguish these newly introduced variable diffusion processes from Lévy statistics. Previous analysis of fluctuations in financial markets appear to contradict the Lévy mechanism.

Throughout the paper, we will relate our results to turbulent flows and financial markets to illustrate implications of our assertions. However, it should be emphasized that our work is a theory of neither of these systems.

\section{MARTINGALE CENTRAL LIMIT THEOREM}

The classical CLT in the context of identical independently distributed events $\left\{\varepsilon_{k}\right\}$ with zero mean and variance $\sigma^{2}$, states that

$$
\frac{1}{\sqrt{n}} \sum_{k=1}^{n} \varepsilon_{k} \rightarrow \mathcal{N}\left(0, \sigma^{2}\right)
$$

as $n \rightarrow \infty$, where $\mathcal{N}\left(0, \sigma^{2}\right)$ denotes a zero-mean normal distribution with variance $\sigma^{2}$. Here the convergence is in distribution; i.e., for each $a$,

$$
\lim _{n \rightarrow \infty} P\left(\frac{1}{\sqrt{n}} \sum_{k=1}^{n} \varepsilon_{k} \leqslant a\right)=\frac{1}{\sqrt{2 \pi} \sigma} \int_{-\infty}^{a} e^{-x^{2} / 2 \sigma^{2}} d x .
$$


The CLT can be generalized for a class of processes, referred to as martingales. We describe the setup only for the case of interest to us.

We consider a random walk (on the real line) starting at $x_{0}=0$, with steps denoted by $\boldsymbol{\varepsilon}=\left(\varepsilon_{1}, \varepsilon_{2}, \ldots\right)$. The step $\varepsilon_{k}$ can depend on its history (i.e., the previous $(k-1)$ steps). The position after $n$ steps is denoted by $x_{n}=\sum_{k=1}^{n} \varepsilon_{k}$. A probability measure $p$ is given on the space of the infinite sequences; $p$ induces a measure $p_{n}\left(\boldsymbol{\varepsilon}^{(n)}\right)$ on the space of first $n$ steps $\boldsymbol{\varepsilon}^{(n)}$. When the context is clear, we denote $p_{n}$ by $p$. If the random variables $\varepsilon_{k}$ are discrete, then $p_{n}$ includes $\delta$-functions.

The conditional probability of the $k$ th step given the history $\boldsymbol{\varepsilon}^{(k-1)}$ is (abusing notation for continuous distributions) defined by

$$
p\left(\varepsilon_{k} \mid \boldsymbol{\varepsilon}^{(k-1)}\right)=\frac{p\left(\boldsymbol{\varepsilon}^{(k)}\right)}{p\left(\boldsymbol{\varepsilon}^{(k-1)}\right)} .
$$

The random variables $\left\{x_{n}\right\}_{n} \geqslant 0$ form a martingale if each increment (or martingale difference) $\varepsilon_{k}$ has zero conditional mean: i.e., if for each $k \geqslant 1$

$$
E\left[\varepsilon_{k} \mid \boldsymbol{\varepsilon}^{(k-1)}\right]=0
$$

for all histories $\boldsymbol{\varepsilon}^{(k-1)}$. (Note that, although this mean value is independent of the history of the walk, the conditional probability density given in Eq. (3) can depend on $\boldsymbol{\varepsilon}^{(k-1)}$.) Finally define the expected value of the location over all $n$-step random walks by

$$
E\left[x_{n}\right]=\int x_{n} d p\left(\boldsymbol{\varepsilon}^{(n)}\right)=\int d \boldsymbol{\varepsilon}^{(n)} x_{n} p\left(\boldsymbol{\varepsilon}^{(n)}\right),
$$

where the second formula is written just to emphasize the variables over which the integration takes place. Denote the corresponding variance by $\operatorname{Var}\left[x_{n}\right]$. We have the following lemma for martingale processes.

Lemma. If $\left\{x_{n}\right\}$ is a martingale process with $x_{0} \equiv 0$, then

(I) $E\left[x_{n}\right]=0$.

(II) $\operatorname{Var}\left[x_{n}\right]=\sum_{k=1}^{n} \operatorname{Var}\left[\varepsilon_{k}\right]$.

These results can be proved inductively using ${ }^{(19)}$

$$
\int d \varepsilon_{n+1} p\left(\varepsilon_{n+1} \mid \boldsymbol{\varepsilon}^{(n)}\right)=1
$$


and

$$
\int d \varepsilon_{n+1} \varepsilon_{n+1} p\left(\varepsilon_{n+1} \mid \boldsymbol{\varepsilon}^{(n)}\right)=E\left[\varepsilon_{n+1} \mid \boldsymbol{\varepsilon}^{(n)}\right]=0
$$

For martingales, Theorem 3.2 of ref. 20 gives a more general form of the CLT. Recall that a sequence $y_{n}$ of random variables is said to converge in probability to a random variable $y$ if for any $\delta>0$, the probability of $\left|y_{n}-y\right|>\delta$ goes to zero as $n \rightarrow \infty$.

Theorem (Martingale central limit theorem) . Suppose that $\varepsilon_{1}, \varepsilon_{2}, \ldots$ are square-integrable martingale differences such that

(1) $\max _{1 \leqslant k \leqslant n}\left(\left|\varepsilon_{k}\right| / \sqrt{n}\right) \rightarrow 0$ in probability,

(2) $\sum_{k=1}^{n} \varepsilon_{k}^{2} / n \rightarrow \eta^{2}$ in probability,

(3) $E\left[\max _{1 \leqslant k \leqslant n}\left(\varepsilon_{k}^{2} / n\right)\right]$ is bounded in $n$,

where the random variable $\eta$ is finite with probability 1 . Then

$$
\frac{1}{\sqrt{n}} \sum_{k=1}^{n} \varepsilon_{k} \rightarrow Z
$$

where the convergence is in distribution (see Eq. (2)), and the random variable $Z$ has characteristic function (i.e., $E[\exp (i t Z)]$ ) given by

$$
E[\exp (i t Z)]=E\left[\exp \left(-\frac{1}{2} \eta^{2} t^{2}\right)\right] \quad \text { for all } t
$$

Observe that the martingale differences $\varepsilon_{k}$ are not required to be independent or to be distributed identically. However, when the conditions of the theorem are satisfied, the distribution of the random variable $u_{n}=\frac{x_{n}}{\sqrt{n}}$ converges to $F(u)$, the distribution of $Z$. We will refer to this property as scalability with scaling index $\zeta=\frac{1}{2}$.

We first provide a necessary and sufficient condition to obtain Gaussian statistics.

Lemma. If the random variables $Z$ and $\eta$ satisfy Eq. (9), then $Z$ is Gaussian if and only if $\eta^{2}$ is a constant.

Indeed, if $\eta^{2}$ is constant, say $\sigma^{2}$, then $Z$ has characteristic function $\exp \left(-\left(\sigma^{2} t^{2}\right) / 2\right)$, and therefore it is normally distributed with variance $\sigma^{2}$. 
Conversely, if $Z$ has mean zero and is a Gaussian with variance $\sigma^{2}$, then its characteristic function equals $\exp \left(-t^{2} \sigma^{2} / 2\right)$. Write $s=\frac{1}{2} t^{2}, g=\eta^{2}$, and define the probability measure $d \widetilde{P}=e^{\sigma^{2}} e^{-g} d P$. Then all moments of $g$ exist with respect to $\widetilde{P}$. The equality

$$
e^{-\sigma^{2} s}=\int e^{-g s} d P
$$

implies, upon differentiating with respect to $s$ and setting $s=1$, that $\widetilde{E}\left[g^{n}\right]=\sigma^{2 n}$ for all $n$. Thus $\eta^{2}$ is constant (Theorem 3.11 of ref. 21).

Next, we identify a set of conditions that gives anomalous statistics for $u_{n}$. Condition (3) of the martingale CLT is satisfied if increments $\varepsilon_{k}$ have a variance bounded uniformly in $n$ (i.e., there exists a $c>0$ such that for all $k, \operatorname{Var}\left[\varepsilon_{k}\right]=E\left[\varepsilon_{k}^{2}\right] \leqslant c$ ). To see this, note that

$$
E\left[\max _{1 \leqslant k \leqslant n}\left(\varepsilon_{k}^{2} / n\right)\right] \leqslant E\left[\sum_{k=1}^{n} \varepsilon_{k}^{2} / n\right]=\frac{1}{n} \sum_{k=1}^{n} \operatorname{Var}\left[\varepsilon_{k}\right] \leqslant c,
$$

Condition (1) is satisfied under the stronger property that there exists $\delta>0$ and $c_{1}>0$ such that for all $k$

$$
E\left[\left|\varepsilon_{k}\right|^{2+\delta}\right] \leqslant c_{1}
$$

This can be seen from

$$
\operatorname{Prob}\left(\max _{1 \leqslant k \leqslant n}\left(\left|\varepsilon_{k}\right| / \sqrt{n}\right)>\beta\right) \leqslant \sum_{k=1}^{n} \operatorname{Prob}\left(\left|\varepsilon_{k}\right| / \sqrt{n}>\beta\right)=\sum_{k=1}^{n} \operatorname{Prob}\left(\left|\varepsilon_{k}\right|>\sqrt{n} \beta\right)
$$

and the fact that

$$
c_{1} \geqslant E\left[\left|\varepsilon_{k}\right|^{2+\delta}\right] \geqslant(\sqrt{n} \beta)^{2+\delta} \operatorname{Prob}\left(\left|\varepsilon_{k}\right|>\sqrt{n} \beta\right) .
$$

Therefore $\operatorname{Prob}\left(\left|\varepsilon_{k}\right|>\sqrt{n} \beta\right) \leqslant c_{1} /(\sqrt{n} \beta)^{2+\delta}$, which implies that

$$
\operatorname{Prob}\left(\max _{1 \leqslant k \leqslant n}\left(\left|\varepsilon_{k}\right| / \sqrt{n}\right)>\beta\right) \leqslant n \frac{c_{1}}{(\sqrt{n} \beta)^{2+\delta}} \rightarrow 0 \quad \text { as } n \rightarrow \infty .
$$


What remains is to find martingales that satisfy condition (2), where $\eta^{2}$ is not a constant. If $\varepsilon_{k}$ 's are independent and identically distributed with finite variance $\sigma^{2}$, then from the classical CLT $\eta^{2}=\sigma^{2}$ in probability. Once $\varepsilon_{k}$ 's are allowed to be history dependent, the conditions for convergence of $\left(\Sigma \varepsilon_{k}^{2} / n\right)$ become non-trivial, as illustrated by the following example: consider a stochastic process with independent steps, consisting of $N_{1}$ steps from a distribution with finite variance $\sigma_{1}^{2}$, followed by $M_{1}$ steps from a distribution with finite variance $\sigma_{2}^{2}$, followed by $N_{2}$ steps from the first process, $M_{2}$ steps from the second, etc. For suitable choices of $N_{1} \ll M_{1} \ll N_{2} \ll M_{2} \ll \cdots, \eta^{2}$ moves between $\sigma_{1}^{2}$ and $\sigma_{2}^{2}$, and fails to converge. Convergence of $\eta^{2}$ requires more stringent conditions on the stochastic process.

For processes introduced in Section 3, the distribution of $\eta^{2}$ is not a $\delta$-function, as shown in Appendix A.

We conclude this section with the following observations. First, we reiterate that once the conditions of the martingale CLT are satisfied, $u_{n}=$ $x_{n} / \sqrt{n}$ converges to a distribution $F(u)$; i.e., the scaling index $\zeta$ is $\frac{1}{2}$. Second, in contrast to Lévy processes, increments $\varepsilon_{k}$ are not independent. Further, for the examples we consider, the $\varepsilon_{k}$ 's satisfy Eq. (10) (at least, according to the numerical simulations). Note however that the conditional variance, $\operatorname{Var}\left[\varepsilon_{n+1} \mid \boldsymbol{\varepsilon}^{(n)}\right]$, is not required to be uniformly bounded.

\section{CONTINUOUS MARKOV PROCESSES}

In order to study continuous processes, divide the interval $t$ into subintervals of $\delta t$ and let $n=t / \delta t$; it is assumed that $\delta t$ is sufficiently large for many martingale increments to occur in this interval. Now, let $\varepsilon_{k}$ 's denote the martingale increments in intervals $\delta t$. In order for the variance of increments in one unit of time to be uniformly bounded, it is necessary and sufficient (see the first Lemma) that $\frac{1}{\delta t} \operatorname{Var}\left[\varepsilon_{k}\right]$ be uniformly bounded; i.e., that $\operatorname{Var}\left[\varepsilon_{k} / \sqrt{\delta t}\right]$ be uniformly bounded. A priori, the limit $Z$ may depend on the particular discretization used. For the examples given below, this is not the case, although we have not been able to derive it analytically as yet.

For the remainder of the paper, we limit considerations to Markov processes; i.e., $p\left(\varepsilon_{k} \mid \boldsymbol{\varepsilon}^{(k-1)}\right)=p\left(\varepsilon_{k} \mid x_{k-1} ;(k-1)\right)$ for each $k$; here, the possible dependence of the probability density on the step number (see Section 2) is denoted explicitly. Markov processes satisfy the master equation $^{(18)}$

$$
W(x ; t+\delta t)=\int d \varepsilon W(x-\varepsilon ; t) p_{\delta t}(\varepsilon \mid(x-\varepsilon) ; t),
$$


where $p_{\delta t}(\varepsilon \mid x ; t)$ denotes the probability density function for an increment $\varepsilon$ to occur in time $\delta t$ beginning from $(x ; t)$. Taylor expanding in the variables $t$ and $x$ about $W(x ; t)$, and noting that $\operatorname{Var}[\varepsilon / \sqrt{\delta t}]$ is bounded, gives the Fokker-Planck equation ${ }^{(17,18,22)}$

$$
\frac{\partial}{\partial t} W(x ; t)=\frac{1}{2} \frac{\partial^{2}}{\partial x^{2}}(D(x ; t) W(x ; t)),
$$

where the diffusion coefficient $D(x ; t)$ is given by

$$
D(x ; t)=\frac{1}{\delta t} \int d \varepsilon \varepsilon^{2} p_{\delta t}(\varepsilon \mid x ; t)=\operatorname{Var}\left[\frac{\varepsilon}{\sqrt{\delta t}} \mid x ; t\right] .
$$

The derivation assumes the martingale condition $E[\varepsilon \mid x ; t]=\int d \varepsilon \varepsilon$ $p_{\delta t}(\varepsilon \mid x ; t)=0$.

Observe next that, since the scaling index $\zeta=\frac{1}{2}$, the probability density for scalable martingales can be written as

$$
W(x ; t)=\frac{1}{\sqrt{t}} F(u),
$$

where $u=x / \sqrt{t}$, and the pre-factor $1 / \sqrt{t}$ has been included in order that $W(x ; t)$ be normalized (i.e., $\int d x W(x ; t)$ be time-independent). Only certain forms of $D(x ; t)$ can be consistent with this requirement. In order to obtain them, change variables so that $D(x ; t)=\bar{D}(u ; t)$. Substituting in the Fokker-Planck equation gives

$$
\frac{\partial}{\partial u}(u F(u))+\frac{\partial^{2}}{\partial u^{2}}(\bar{D}(u ; t) F(u))=0
$$

which can be integrated to

$$
u F(u)+\frac{\partial}{\partial u}(\bar{D}(u ; t) F(u))=c_{1}(t)
$$

Here $c_{1}(t)$ is the "constant" of integration. Integrating a second time gives

$$
\bar{D}(u ; t)=-\frac{1}{F(u)} \int_{-\infty}^{u} d v v F(v)+\frac{1}{F(u)}\left(c_{1}(t) u+c_{2}(t)\right) .
$$

where $c_{2}(t)$ is the second constant of integration. 
Examples. The Gaussian distribution $F(u)=\exp \left(-\frac{1}{2} u^{2}\right)$ corresponds to $\bar{D}(u ; t)=1+\left(u c_{1}(t)+c_{2}(t)\right) \exp \left(\frac{1}{2} u^{2}\right)$. The exponential distribution $F(u)=\exp (-|u|)$ corresponds to a diffusion coefficient $\bar{D}(u ; t)=(1+|u|)+$ $\left(u c_{1}(t)+c_{2}(t)\right) \exp (-|u|)$.

Note that the terms in $\bar{D}(u ; t)$ that contain $c_{1}(t)$ and $c_{2}(t)$ do not change the form of $W(x ; t)$. Hence, they will not be considered in the remainder of the paper; i.e., only the $t$-independent part of $\bar{D}(u ; t)$, henceforth denoted $D(u)$, will be considered.

Conversely, if the diffusion coefficient $D(u)$ is given, Eq. (15) can be integrated to give

$$
F(u)=\frac{1}{D(u)} \exp \left(-\int^{u} d v \frac{v}{D(v)}\right)\left[a_{1} \int^{u} d v \exp \left(\int^{v} d w \frac{w}{D(w)}\right)+a_{2}\right],
$$

where $a_{1}$ and $a_{2}$ are constants of integration. If $D(u)$ is symmetric under reflections about the origin and the process begins at $x_{0}=0$ then $F(u)$ is symmetric; ${ }^{(23)}$ consequently $a_{1}=0$, as can be seen from the anti-symmetry of the left-hand side of Eq. (16). Then,

$$
F(u)=\frac{1}{D(u)} \exp \left(-\int^{u} d v \frac{v}{D(v)}\right)
$$

The form of $F(u)$ for selected diffusion rates is given next. As mentioned in Section 2, although $\operatorname{Var}\left[\varepsilon_{n} / \sqrt{\delta t}\right]$ for each $n$ is uniformly bounded, the conditional variance $\operatorname{Var}\left[\varepsilon_{n} / \sqrt{\delta t} \mid x ; t\right]$ of the martingale differences, given by $D(u)$, is not required to be bounded with respect to $u$.

\section{Examples.}

(I) $D(u)=1 \longrightarrow F(u)=\exp \left(-\frac{1}{2} u^{2}\right)$,

(II) $D(u)=1+\alpha|u| \longrightarrow F(u)=\exp \left(-\frac{|u|}{\alpha}\right) /(1+\alpha|u|)^{\left(1-\alpha^{-2}\right)}$,

(III) $D(u)=1+|u| \longrightarrow F(u)=\exp (-|u|)$,

(IV) $D(u)=\left(1+\alpha u^{2}\right) \longrightarrow F(u)=\left(1+\alpha u^{2}\right)^{-(1+(1 / 2 \alpha))}$.

Thus, suitable choices of $D(u)$ can give exponential or power-law behavior in $F(u)$. Note that, in the final example $\alpha<1$ is needed in order for the condition (10) to be satisfied. 
We have confirmed numerically that stochastic dynamics with diffusion coefficients given in these examples give probability density functions consistent with the analytically derived expressions. These computations were conducted by integrating the (zero-drift) Langevin equation $d X=$ $[D(X(t) ; t)]^{1 / 2} \mathcal{N}(0, d t) .{ }^{(24)}$ The integrations are done using Ito calculus; i.e., it is assumed that each step in the integration consists of a large number of stochastic increments and that variations in $D(X(t) ; t)$ during the interval can be ignored. Consequently, the deviations in a time interval $\delta t$ follows $\mathcal{N}(0, \sqrt{D} \delta t)$.

\section{DISTINGUISHING BETWEEN LÉVY AND VARIABLE DIFFUSION PROCESSES}

Given a stochastic process $\left\{\varepsilon_{k}\right\}$ such that $x_{n}$ has a scalable, non-normal distribution, is it possible to determine if Lévy or variable diffusion processes are the more likely source of the dynamics? More precisely, is it possible to eliminate one of the scenarios as the underlying cause of the observed stochastic dynamics? One possible criterion is to test if the variance of the stochastic process is finite (variable diffusion) or infinite (Lévy). However, it is difficult to make this determination from a finite time series. An alternative is to use the fact that while successive movements of a Lévy process are independent, those in the variable diffusion case depend on the location and time of the walk. For example, if $D(u)$ increases with $|u|$ (as in the examples above), then large movements are likely to leave $x_{n}$ (and hence $D(u)$ ) large; consequently, a large fluctuation can generally be expected to be followed by additional (positive or negative) large increments. Lévy processes with independent increments will not exhibit such correlations. Dynamics of Lévy and variable diffusion processes, shown in Fig. 1, illustrates the difference.

Thus, one may consider distinguishing variable diffusion and Lévy processes using the auto-correlation function of a time series. However, since the process is martingale, the auto-correlation will vanish. On the other hand, auto-correlation function of $\left\{\varepsilon_{k}^{2}\right\}^{(n)}$ will only vanish for the Lévy case. Specifically, for a random time series of length $n$, we use

$$
\mathcal{C}(m ; n) \equiv \frac{1}{\operatorname{Var}\left[\varepsilon^{2}\right]}\left\langle\left(\varepsilon_{k}^{2}-\left\langle\varepsilon^{2}\right\rangle\right)\left(\varepsilon_{k+m}^{2}-\left\langle\varepsilon^{2}\right\rangle\right)\right\rangle,
$$

where $\langle$.$\rangle denotes the average over k$. For Lévy processes, it is found numerically that for $m>0, \mathcal{C}(m ; n)$ decreases to zero as $n$ increases, while for variable diffusion processes with $D(u)=1+|u|$, it is found to decay as 

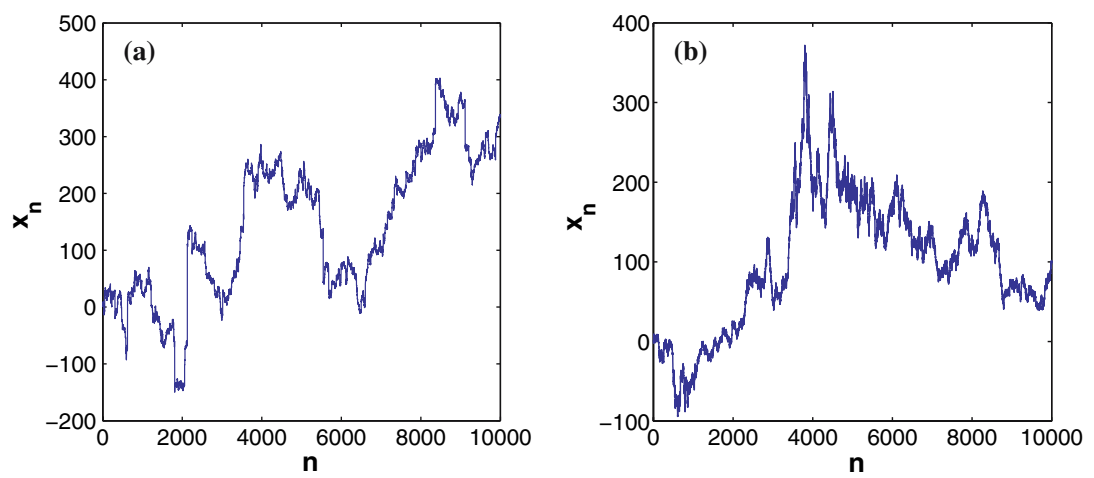

Fig. 1. Examples of 10,000 steps from a (a) Lévy distribution with $\zeta=2 / 3$ and (b) variable diffusion process with $D(u)=2\left(1+u^{2}\right)$. Unlike in (a), a large fluctuation in (b) is generally followed by movements with higher amplitude.

$\exp (-\alpha m / n)$; the $n$-dependence implies that a longer series contains larger fluctuations.

For fluctuations in financial markets, $\mathcal{C}(m ; n)$ is known to exhibit a slow decay with $m .^{(25)}$ This phenomenon, referred to as "clustering of volatility," suggests that Lévy processes are unlikely to be the source of scalable non-Gaussian distributions in financial markets.

\section{DISCUSSION}

The theory we have presented is not merely a reformulation where an observed scalable probability density function $W(x ; t)$ is recast into a suitably chosen diffusion coefficient $D(x ; t)$. Rather, it introduces a new class of stochastic dynamics. Unlike Lévy processes, the increments considered in our work, although Markovian, are not independent. In addition, they have finite variances. The scaling index for scalable diffusion processes takes a unique value $\zeta=\frac{1}{2}$. The probability density function $W(x ; t)$ for continuous time stochastic dynamics takes the form $\frac{1}{\sqrt{t}} F(u)$ and satisfies the Fokker-Planck equation. The diffusion coefficient can be chosen to be a function of $u$, and there is a correspondence between $F(u)$ and the diffusion coefficient $D(u)$.

The fact that successive events are independent in Lévy processes and only martingales in our variable diffusion processes implies that dynamics can be used to identify which model is more suitable to represent a given time series of stochastic events. We propose the use of the auto-correlation of $\varepsilon_{k}^{2}$,s as such a test. Previous studies of financial markets suggest 
that they consist of increments that are not independent, and hence suggest that independent Lévy processes are unlikely to be the correct explanation for the observed non-Gaussian probability density functions. ${ }^{(25)}$

The need for $x$-dependent diffusion coefficients implies that the stochastic dynamics is not invariant under translations in $x$. In particular, for the examples given earlier, the origin is both the starting point of the walk as well as the location where $D(x ; t)$ is minimized. In financial markets, one does expect any sudden large fluctuation in the price of a stock to be followed by a period of high anxiety in the part of traders; consequently the stock can be expected to trade at a significantly higher rate. This is equivalent to an increase in the diffusion rate. However, if the price of the stock settles at this new value, it is likely that the location of the minimum in $D(x ; t)$ will move towards it. Thus, a more realistic model of financial markets would involve a coupled variation of the price of the stock and the location of the minimum of the diffusion coefficient. ${ }^{(26)}$

A time-dependent, but $x$-independent drift $\mu(t)$ of the stochastic process can be introduced by including a "drift" term $-\mu(t) W(x ; t)$ on the right side of the Fokker-Planck equation. ${ }^{(18)}$ Redefining $u$ to be $\frac{1}{\sqrt{t}}\left(x-\int^{t} \mu(s) d s\right)$ gives Eq. (12), and the rest of the analysis presented here follows.

Finally, one may inquire if the martingale CLT can be generalized to include processes whose distributions do not scale as $n^{1 / 2}$; i.e., the variable $Z$ in Eq. (8) is the limit of $\sum \varepsilon_{k} / n^{\xi}$, where $\xi$ is not necessarily $1 / 2$. In particular, can Lévy processes fit into such a description. A related question is if and under what conditions do Lévy processes with memory give selfsimilar distributions. We are currently working towards addressing these issues.

\section{APPENDIX A: ANOMALOUS MARTINGALE PROCESSES}

When the diffusion coefficient is a function of $u$, the martingale sums may fail to have a normal distribution. We have chosen processes where $E\left[\left|\varepsilon_{k}\right|^{2+\delta}\right]$ is uniformly bounded, so that conditions (1) and (3) of the martingale CLT are satisfied. Hence, the random variable $Z$ is not distributed normally because $(1 / n) \sum \varepsilon_{k}^{2}$ does not approach a constant (in probability) for large $n$. We illustrate this failure with two examples of discrete random walks.

The distribution of $\eta^{2}$ for a finite-step martingale with $D(u)=1+$ $|u|$ is shown in Fig. 2(a). Since $D(u) \geqslant 1$ for all $u, \eta^{2}$ is non-vanishing only when the argument is larger than 1 , where it decays exponentially. As expected from the analysis, $F(u)$ is found to be $\frac{1}{2} \exp (-|u|)$. 

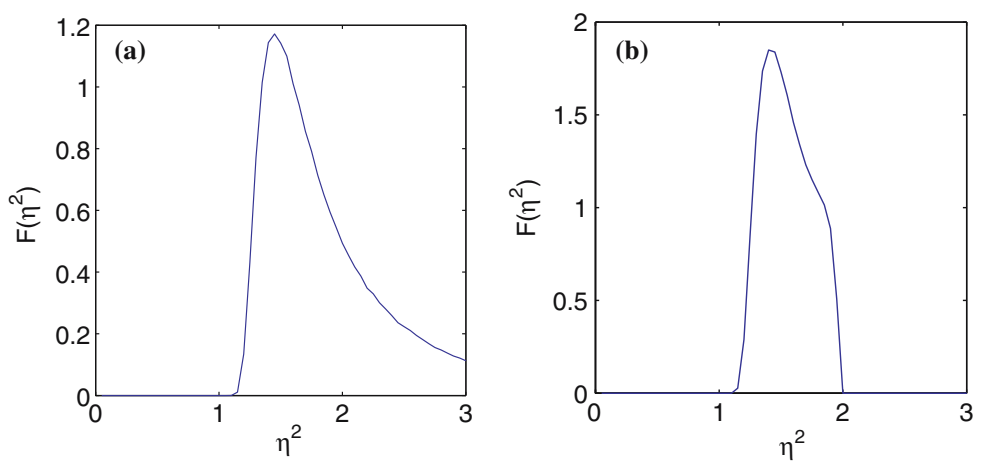

Fig. 2. The density function $F$ of $\eta^{2}=\lim _{n \rightarrow \infty} \frac{1}{n} \sum_{1}^{n} \varepsilon_{k}^{2}$ for random walks with (a) $D(u)=$ $1+|u|$, and (b) $D(u)=1+\tanh (|u|)$. The fact that they are not $\delta$-functions implies that $\lim \left(x_{n} / \sqrt{n}\right)$ is not Gaussian, see Section 2.

Next, consider a martingale with $D(u)=(1+\tanh |u|)$. For a fixed $t, D(u)$ varies between 1 and 2 , and for a fixed $x$, it reduces to 1 with increasing $t$. The histogram of $\eta^{2}$, computed numerically for a set of 100,000 random walks of length 100,000 , converges to the function shown in Fig. 2(b). Since $1 \leqslant D(u) \leqslant 2, \eta^{2}$ is non-zero only in the interval $[1,2]$. The corresponding probability density function $W(x ; t)$ has the form $\frac{1}{\sqrt{t}} F(u)$, but $F(u)$ is not Gaussian. In contrast, if the diffusion coefficient is chosen to be $(1+\tanh |x|)$ or $(1+\tanh (1 / \sqrt{t})), \eta^{2}$ is found to be constant, and $W(x ; t)$ is found to approach a Gaussian.

\section{ACKNOWLEDGMENTS}

The research of GHG is partially supported by the NSF Grant PHY0201001 and a grant from the Institute of Space Science Operations at the University of Houston (GHG). The research of M. Nicol and A. Török was supported in part by NSF Grant DMS-0244529.

\section{REFERENCES}

1. F. Heslot, B. Castaing, and A. Libchaber, Phys. Rev. A 36:5870 (1987).

2. B. Castaing, G. Gunaratne, F. Heslot, A. Libchaber, L. P. Kadanoff, S. Thomae, X. Wu, S. Zaleski, and G. Zanetti, J. Fluid. Mech. 204:1 (1989).

3. X. Z. Wu, L. P. Kadanoff, A. Libchaber, and M. Sano, Phys. Rev. Lett. 64:2140 (1990).

4. T. H. Solomon and J. P. Gollub, Phys. Rev. Lett. 64:2382 (1990).

5. T. Takashita, T. Segawa, J. A. Glazier, and M. Sano, Phys. Rev. Lett. 76:1465 (1996).

6. P. Embrechts, C. Klüppelberg, and T. Milkoch, Modelling Extreme Events (Springer, Berlin, 2003). 
7. B. B. Mandelbrot, J. Bus. 36:394 (1963).

8. R. N. Mantegna and H. E. Stanley, Nature 376:46 (1995); Nature 383:587 (1996).

9. R. Friedrich, J. Peinke, and Ch. Renner, Phys. Rev. Lett. 84:5224 (2000).

10. A. Arneodo, J.-F. Muzy, and D. Sornette, European Phys. J. B 2:277 (1998).

11. M. M. Dacorogna, R. Gencay, U. Müller, R. B. Olsen, and O. V. Pictet, An Introduction to High-Frequency Finance (Academic Press, San Diego, 2001).

12. J. L. McCauley and G. H. Gunaratne, Physica A 329:178-198 (2003).

13. J. Klafter, M. F. Schesinger, and G. Zumofen, Physics Today, February 1996, page 33; B. D. Hughes, M. F. Schlesinger, and E. W. Montroll, Proc. Natl. Acad. Sci. 78:3287 (1981).

14. J.-P. Bouchaud and A. Georges, Phys. Rep. 195:127 (1990).

15. T. H. Solomon, E. R. Weeks, and H. L. Swinney, Phys. Rev. Lett. 71:3975 (1993).

16. J. Peinke, F. Böttcher, and St. Barth, Ann. Phys. 13:450 (2004).

17. A. D. Fokker, Ann. d. Physik 43:812 (1914); M. Planck, Sitz. der preuss. Akad. p. 324 (1917).

18. S. Chandrasekar, Rev. Mod. Phys. 15:1 (1943).

19. These properties actually follow from $E\left[x_{n}\right]=E\left[x_{n-1}\right]$, and $E\left[x_{n} \varepsilon_{n+1}\right]=$ $E\left[x_{n} E\left[\varepsilon_{n+1} \mid \boldsymbol{\varepsilon}^{(n)}\right]\right]=0$, for any martingale.

20. P. Hall and C. C. Heyde, Martingale Limit Theorem and its Application (Probability and Mathematical Statistics, Academic Press, 1980).

21. R. Durrett, Probability: Theory and Examples, 2nd ed. (Duxbury Press, 1996).

22. S. Maslov and Y.-C. Zhang, Physica A 262:232 (1999).

23. It is possible that the symmetric $F(u)$ is unstable, and the stable distributions consists of a pair of functions related by reflectional symmetry. In the examples given here, the symmetric $F(u)$ is found to be the solution of the Langevin equation for motion starting from the origin.

24. D. T. Gillespie, Markov Processes: An Introduction for Physical Scientists (Academic Press, San Diego, 1992).

25. R. Cont, M. Potters, and J.-P. Bouchaud, Scaling in stock market data: stable laws and beyond, in "Scale invariance and Beyond," Proc. of the CNRS workshop on scale invariance, B. Dubrulle, F. Graner, and D. Sornette eds. (Springer, Berlin, 1997).

26. A. L. Alejandro-Quiñones, K. E. Bassler, M. Field, J. L. McCauley, M. Nicol, I. Timofeyev, A. Török, and G. H. Gunaratne, A Theory of Fluctuations in Stock Prices, to appear in-Physica A (2005). 